\title{
Second primary double carcinomas of the residual cervical esophagus and the gastric tube after thoracic esophagectomy
}

\author{
Yoshifumi Ikeda, MD, Shoichi Tobari, MD, Masanori Niimi, MD, PhD, Susumu Kodaira, MD, and Kota Okinaga, MD, \\ Tokyo, Japan
}

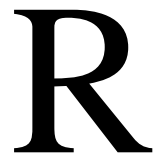

ecent significant advances in therapy for esophageal carcinoma have contributed to long-term survival, with some patients having secondary and multiple malignancies. Patients with squamous cell esophageal carcinoma are generally older, smokers, and alcohol drinkers, and they face a high risk of development of other tumors, such as head and neck carcinoma. ${ }^{1,2}$ In the procedure of subtotal esophagectomy, a small segment of the proximal esophagus is left to form the anastomosis with the substitution. Therefore there is a risk of development of a second neoplasm in the proximal remnant esophagus in patients who survive the first esophageal carcinoma. Secondary carcinoma in a reconstructed gastric tube after esophagectomy has also received much attention because the incidence of gastric carcinoma is more frequently than in any other organ. ${ }^{3}$ In this report we describe second primary double carcinomas of the residual cervical esophagus and the gastric tube after thoracic esophagectomy.

\section{Clinical Summary}

A 72-year-old man was admitted to our department with persistent midthoracic pain, mild dysphagia, and a weight loss of $5 \mathrm{~kg}$ during the previous 3 months. He had smoked 20 cigarettes a day for 40 years but had no respiratory symptoms. An upper gastrointestinal endoscopic study revealed a friable tumor $5 \mathrm{~cm}$ in length in the middle esophagus. On February 21, 1994, the patient underwent a subtotal esophagectomy and lymphadenectomy through a right thoracotomy and laparotomy, and intrathoracic eosphagogastrostomy was performed. Microscopically, the esophageal tumor was a well-differentiated squamous cell carcinoma invading the adventitia with lymph node metastasis (pT3 N1 M0, stage III). The patient's postoperative recovery was uneventful, and he was discharged without any complications.

The patient reported difficulty in swallowing 7 years after the initial operation. An upper gastrointestinal barium-contrast radiographic series revealed a $3 \times 2-\mathrm{cm}$ elevated lesion in the residual cervical esophagus near the esophageal orifice (Figure 1, $A$ ) and a $4 \times 3$-cm elevated lesion in the middle third of the gastric tube

From the Departments of Surgery, Teikyo University School of Medicine, Tokyo, Japan.

Received for publication Sept 4, 2002; accepted for publication Oct 8, 2002.

Address for reprints: Yoshifumi Ikeda, MD, Department of Surgery, Teikyo University School of Medicine, 2-11-1 Kaga, Itabashi-ku, Tokyo 173-8605, Japan (E-mail: yikeda@med.teikyo-u.ac.jp).

J Thorac Cardiovasc Surg 2003;125:1561-2

Copyright $\odot 2003$ by The American Association for Thoracic Surgery $0022-5223 / 2003 \$ 30.00+0$

doi:10.1016/S0022-5223(03)00052-7
(Figure 1, B). An upper gastrointestinal endoscopic study revealed a 2-cm elevated tumor with severe esophagitis and a 3-cm elevated tumor with ulceration in the gastric tube. Histologic study of the biopsy specimens showed squamous cell carcinoma in the residual cervical tumor and adenocarcinoma in the tumor of the gastric tube. On March 21, 2001, the patient underwent larynx-preserving resection of the residual cervical esophagus and the gastric tube with cervicotomy, sternotomy, and laparotomy. The cephalic cut line of the esophagus was determined on the basis of the safety margin from the carcinoma after opening the esophageal wall. The right colon was mobilized and then pulled through the cervical incision through the posterior mediastinal route. The anastomosis between the cervical esophagus and the ileum was performed in an end-to-side fashion with the layer-to-layer method. After that an end-to-side colonojejunostomy and an end-to-end ileocolonostomy was created with the Gambee method. The postoperative course was uneventful. Postoperative gastrointestinal studies revealed no anastomotic leakage. Histopathologic examination of the resected specimen confirmed that the esophageal tumor was a moderately differentiated squamous cell carcinoma invading the submucosal layer without lymph node metastasis (pT1 N0 M0, stage I), and the tumor of the gastric tube was a moderately differentiated adenocarcinoma invading the muscularis propria without any lymph node metastasis (pT2 N0 M0, stage IB). The patient's life returned to normal. No recurrent disease, including that at the cephalic enteric anastomotic site, was detected 18 months after surgery.

\section{Discussion}

It is not uncommon for patients with esophageal carcinoma to have multiple primary carcinomas in the stomach or the head and neck region. ${ }^{1-3}$ According to a number of recent reports, about $20 \%$ of patients with thoracic esophageal carcinoma have multiple primary lesions in the esophagus, ${ }^{2}$ and gastric carcinoma is the most common primary carcinoma associated with esophageal carcinoma. ${ }^{3}$ Diagnostic and therapeutic advances for esophageal carcinoma have contributed to good outcomes with respect to patients' survival. Because of this, the incidence of carcinoma in the gastric tube or the residual esophagus has increased, and it is reported in the literature to be $0.2 \%$ to $1.0 \% .^{3-5}$ However, double second primary carcinomas in the remaining esophagus and the gastric tube are very rare. In our case, the double second lesions were actually metachronous primary lesions, because more than 6 years had elapsed after esophagectomy, the cervical lesion was at a relatively early stage and had an epithelial component, and the lesion of the gastric tube was a moderately differentiated adenocarcinoma.

When the second primary carcinoma is early stage, endoscopic mucosal resection is the best treatment choice. Although surgical resection of gastric tube carcinoma or residual esophageal carci- 

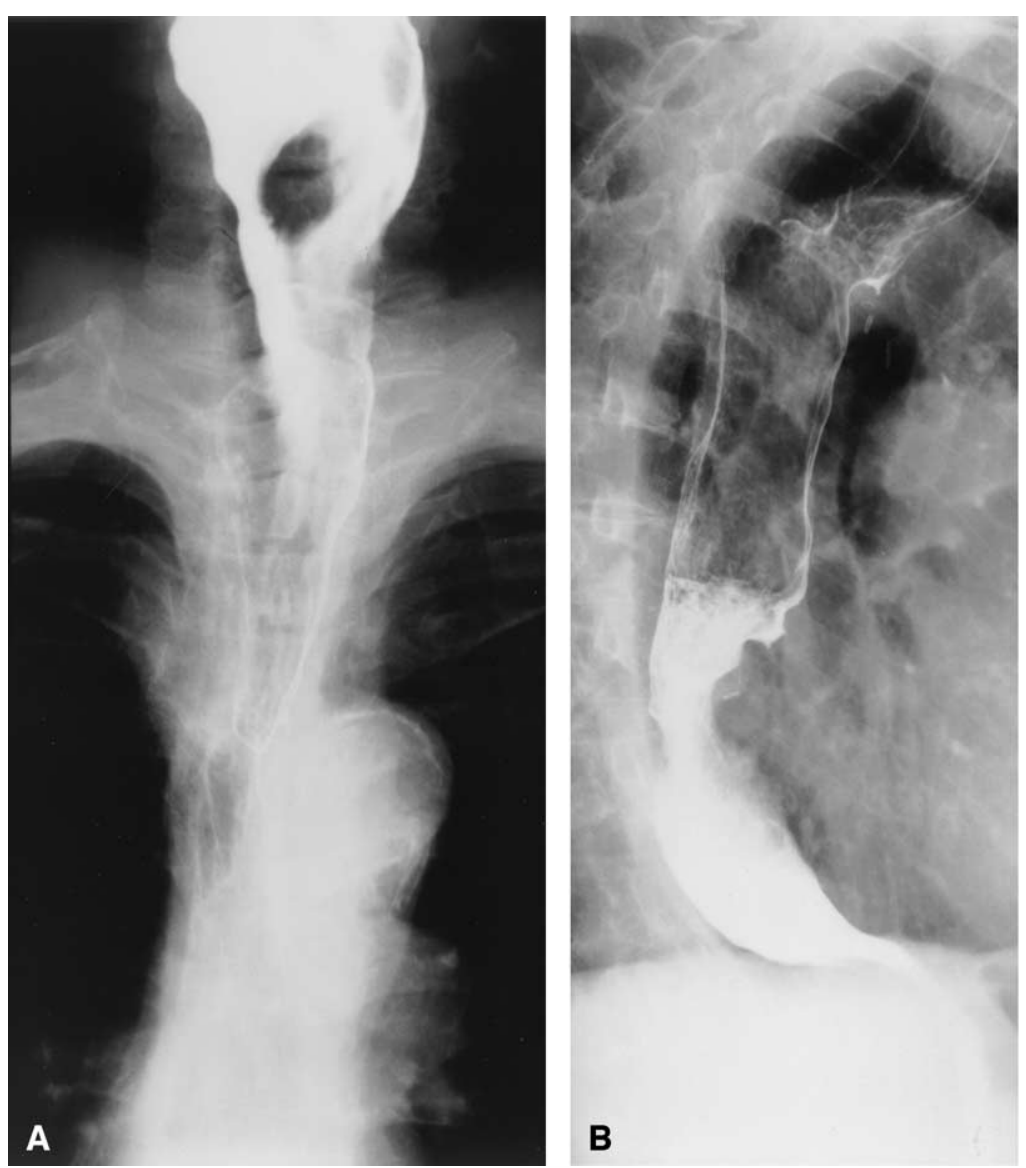

Figure 1. A, Preoperative gastrointestinal barium study reveals small protruding lesion in cervical esophagus near esophageal orifice. $B$, Preoperative gastrointestinal barium study reveals elevated lesion with ulceration in middle third of gastric tube.

noma after esophagectomy places a heavy burden on the patient and carries high morbidity and mortality, surgical treatment is necessary for curative resection of advanced carcinomas. In particular, combined resection of the larynx is necessary for the residual cervical esophageal carcinoma that invades the esophageal orifice. In our surgical procedure, the cephalic cut line of the esophagus was set squarely at the lesion, and a larynx-preserving resection was performed. Because loss of voice results in severe inconvenience for the patient, our procedure is acceptable as treatment for high cervical esophageal carcinoma limited to the submucosal layer.

Early detection of the tumor is necessary for good outcomes of these diseases. Annual endoscopic examination of the residual esophagus and the gastric tube is recommended for patients who have undergone esophagectomy.

\section{References}

1. Tachimori Y, Watanabe H, Kato H, Ebihara S, Ono I, Nakatsuka T, et al. Treatment for synchronous and metachronous carcinomas of the head and neck and esophagus. J Surg Oncol. 1990;45:43-5.

2. Mizobuchi S, Kato H, Tachimori Y, Watanabe H, Yamaguchi H, Itabashi M. Multiple primary carcinoma of the oesophagus. Surg Oncol. 1993;2:249-53.

3. Koide N, Yazawa K, Koike S, Adachi W, Amano J. Oesophageal cancer associated with other primary cancers: a study of 31 patients. $J$ Gastroenterol Hepatol. 1997;12:690-4.

4. Souquet JC, Berger F, Bonvoisin S, Partensky C, Boulez J, Descos F, et al. Esophageal squamous cell carcinoma associated with gastric adenocarcinoma. Cancer. 1989;63:786-90.

5. Kitamura K, Kuwano H, Matsuda H, Mori M, Sugimachi K. Secondary early carcinoma in a reconstructed gastric tube after subtotal esophagectomy for early carcinoma of the esophagus. Hepatogastroenterology. 1993;40:44-7. 